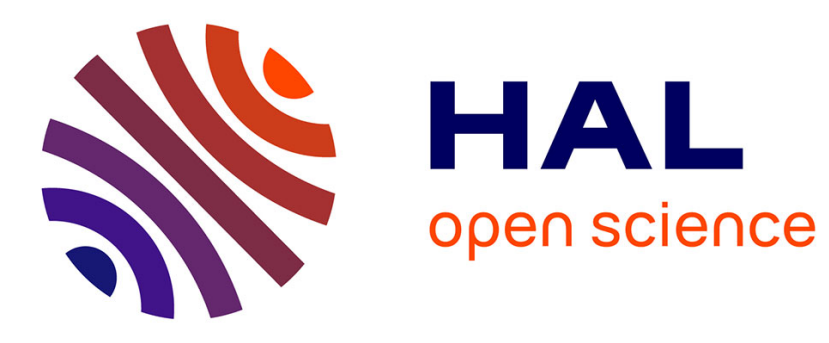

\title{
Lower Hybrid Range Cold Magnetized Plasma Modelling in ANSYS HFSS
}

Julien Hillairet, Riccardo Ragona, Laurent Colas, Walid Helou, Frédéric Bocquet

\section{- To cite this version:}

Julien Hillairet, Riccardo Ragona, Laurent Colas, Walid Helou, Frédéric Bocquet. Lower Hybrid Range Cold Magnetized Plasma Modelling in ANSYS HFSS. Fusion Engineering and Design, 2019, 10.1016/j.fusengdes.2019.02.108 . cea-01889312v2

\section{HAL Id: cea-01889312 https://hal-cea.archives-ouvertes.fr/cea-01889312v2}

Submitted on 7 Apr 2019

HAL is a multi-disciplinary open access archive for the deposit and dissemination of scientific research documents, whether they are published or not. The documents may come from teaching and research institutions in France or abroad, or from public or private research centers.
L'archive ouverte pluridisciplinaire HAL, est destinée au dépôt et à la diffusion de documents scientifiques de niveau recherche, publiés ou non, émanant des établissements d'enseignement et de recherche français ou étrangers, des laboratoires publics ou privés. 


\title{
Lower Hybrid Range Cold Magnetized Plasma Coupling in ANSYS HFSS
}

\author{
Julien Hillairet ${ }^{\mathrm{a}, *}$, Riccardo Ragona ${ }^{\mathrm{b}}$, Laurent Colas $^{\mathrm{a}}$, Walid Helou ${ }^{\mathrm{c}}$, Frédéric Bocquet ${ }^{\mathrm{d}}$ \\ ${ }^{a}$ CEA, IRFM, F-13108 Saint Paul-lez-Durance, France \\ ${ }^{b}$ Laboratory for Plasma Physics, Royal Military Academy, (LPP-ERM/KMS), BE-1000, Brussels, Belgium \\ ${ }^{c}$ ITER Organization, Route de Vinon-sur-Verdon, 13115 St Paul-lez-Durance, France \\ ${ }^{d}$ ANSYS France
}

\begin{abstract}
The coupling between cold magnetized plasmas with Lower Hybrid Resonance Frequency antennas is generally addressed using specifically developed codes. These antenna coupling codes often approximate the plasma to a surface impedance described by a 1D half infinite models and antennas either with simplified 2D dimensions or from 3D CAD models conversion. Such approaches add an additional step of model approximation/conversion which is not convenient to rapidly assess impact of geometry changes on coupling performances. In this work, we assess the ability of using ANSYS HFSS to describe the usual range of experimental magnetized cold plasma inhomogeneous parameters facing LHRF antennas, away from resonances, in order to guide the RF designer during the design phase of an antenna. The coupling calculations of Lower Hybrid Resonance Frequency antennas are performed and successfully benchmarked with the fast coupling codes ALOHA on inhomogeneous cold magnetized plasmas. Good agreements are obtained when the boundary conditions in the full-wave modelling are properly handled. Practical advices and limitations are given in order to define correctly these boundary conditions and insure correct results.
\end{abstract}

Keywords: Lower Hybrid, LH, LHRF, LHCD, Cold Plasma, Finite Elements

\section{Introduction}

Achieving the plasma temperature expected for nuclear fusion requires external heating devices, such as dedicated Radio-Frequency systems. Dimensions, power level and manufacturing cost which are at stake make it impossible to build scaleone mock-up during design and prototyping phases. If only for this reason, modelling the electromagnetic interactions between magnetized plasmas and Radio-Frequency antennas is mandatory for nuclear fusion research. In this paper, we focus only on the RF coupling performances of these antenna to the tokamak edge plasma and not into the wave absorption mechanisms as we assume here that all the power is absorbed into the plasma core.

During the last two decades, the availability of $\mathrm{RF}$ full wave software eased the design of RF antennas, such as Ion Cyclotron (ICRF) and Lower

\footnotetext{
*Corresponding author

Email address: julien.hillairet@cea.fr (Julien Hillairet)

Preprint submitted to Fusion Engineering and Design
}

Hybrid Resonance Frequency systems (LHRF). As both software and hardware progressed, modelling has become more and more realistic, reducing the gap between CAD and RF models and accelerating the necessary feedback between mechanical and RF engineers. Nowadays, thermal or mechanical loads can be provided directly from the results of RF simulations inside integrated work flows, which make design phases faster.

When the magnetic field is oriented along the $z$ axis, the plasma facing the antennas can be described in the cold-plasma approximation by the following relative permittivity tensor in the $e^{j \omega t}$ time-harmonic convention:

$$
\varepsilon_{r}=\left(\begin{array}{ccc}
S & j D & 0 \\
-j D & S & 0 \\
0 & 0 & P
\end{array}\right)
$$

where the parameters $S, D, P$ are described in [1] and depends of the RF frequency, the plasma species and the confining magnetic field. A decade ago, antenna to plasma coupling modelling with commercial full-wave tools was not possible unless

January 25, 2019 
some severe simplifications, as they used to not support anisotropic and inhomogeneous dielectric media. Community codes such as OLGA [2] or ALOHA [3] and TOPLHA [4] for LHRF were developed specifically for that purpose. While these tools are generally faster than full-wave modelling since they solve part of the problem in the spectral domain, their Doppelgänger is to assume slab plasma and eventually simplified antenna geometries to keep an analytical formulation of the problem. When dealing with detailed and curved antennas, poloidal and toroidal plasma curvatures or wave scattering by plasma inhomogeneities, 3D numerical approaches become mandatory.

Since the last decade, full wave codes such as COMSOL, Microwave CST or ANSYS HFSS for example, have been able to define propagating medium as anisotropic tensor with eventually negative or complex permittivity, spatial and frequency dependences. This ability allowed coupling calculations of the C-Mod LH launcher[5]. Recently, the open-source initiative [6] allows modelling RF waves propagation in edge and core plasmas. In all cases, a difficulty arises in the model setup if geometry is partially taken into account to save resources: in this case, boundary conditions at the edges of the plasma domain need to be defined. Indeed, default radiation or absorbing boundary conditions such as Perfectly Matched Layer (PML) shipped in all these software are not designed to be used directly on the anisotropic material boundaries but instead on isotropic mediums. For antenna to plasma coupling, the plasma medium in which the antenna radiate is at the contrary considered as an semi-infinite medium and should be thus defined with attached ideal absorbing boundary conditions. Specific PML for anisotropic materials such as a magnetized cold plasma have been used in [7] and mathematically studied in [8].

Thanks to the collaboration between CEA, ITER and ANSYS, the RF modelling software ANSYS HFSS supports inhomogeneous anisotropic medium since 2016. The purpose of the present work is not to produce a rigorous approach of the coupling simulation or to model the wave absorption mechanism in the plasma or non-linear effects such as sheaths on the antenna boundaries. The motivation is to assess the ability of HFSS to describe the usual range of experimental magnetized cold plasma inhomogeneous parameters facing LHRF (sec.2) antennas, away from resonances, in order to guide the $\mathrm{RF}$ designer during the design phase of an antenna.
Due to space constraint, this paper focuses only on the LHRF case, but the treatment of ICRF cases is similar and a future paper will be dedicated to the ICRF topic.

\section{LHRF Coupling}

In the Lower Hybrid Current Drive range of frequencies $(2-6 \mathrm{GHz})$ and edge plasma electron densities in current tokamak experiments $\left(n_{e}=\right.$ $10^{17}-10^{18} \mathrm{~m}^{-3}$ ), the cold plasma dielectric tensor is mostly diagonal $\varepsilon_{r} \approx(1,1, P)$ with $\varepsilon_{r, z z}=$ $P \approx 1-\frac{\omega_{p e}^{2}}{\omega^{2}}=1-\frac{n_{e}}{n_{c}}$ where $\omega_{p e}$ and $\omega$ are respectively the plasma and the RF angular frequency and $n_{c}=\frac{\omega^{2} m_{e} \varepsilon_{0}}{e^{2}}$ the slow-wave cut-off density. In practice, operational plasma parameters lead to $P \in[-10,0]$. Thus, as $n_{e}$ increases, the required mesh length in the full wave simulation should decrease accordingly to match the decrease of the skin depth which depends of both permittivity (and conductivity).

In HFSS, using standard PML around such a medium does not give satisfactory results and one should consider instead developing its own PMLs as done in [7]. A workaround consists in adding progressively artificial losses, also known as "adiabatic absorbers"[9]. If the waves are sufficiently attenuated before they reach the propagation domain boundaries, the edge boundaries can be set as perfect electric conductors. In order not to interfere too much with the coupling results, a non-lossy region is made in front of the antenna (Figure 1). A power law of increasing conductivity has been used to attenuate the field, as $\sigma(x)=\alpha_{x}\left(x-x_{0}\right)^{2}$ if $x>x_{0}$ and zero otherwise, with $\alpha_{x}$ a scaling parameter and $x_{0}$ the location of the lossy region limit. Similar expressions are used in direction $y$ and $z$ for $\sigma(y)$ and $\sigma(z)$. The function $\sigma(x)$ can be directly set-up in the material editor or as a global parameter, paying attention that the spatial parameters $(x, y, z)$ are the one of the local material coordinates.

As a rule of thumb, a toroidal region at least four times larger than the antenna (toroidal) width and a radial depth at least as large as the antenna (toroidal) width are sufficient, as long as the conductivity law in the radiating region absorb enough power. Without magnetic field tilt angle and without phasing law between waveguide rows, propagation is mainly restricted to the $\mathrm{XZ}$ plane and thus the poloidal volume has low influence. A poloidal 
size of less than twice the height of the antenna is sufficient. In practice, a parametric scan should be first performed on the magnitude of the conductivity law $\alpha$ until convergence is obtain on the result of interest, for example on the reflection coefficients of the antenna.

In the example presented in figure 1, a 3D LHRF antenna made of six waveguides $(76 \mathrm{~mm}$ heigh in poloidal direction, $8.5 \mathrm{~mm}$ width in toroidal direction and spaced by $2 \mathrm{~mm}$ and excited at $3.7 \mathrm{GHz}$ ) is facing a small vacuum gap (few millimetres of less) before a linearly increasing density plasma described by $n_{e}(x)=n_{e 0}\left(1+\frac{x-d_{\text {gap }}}{\lambda_{n}}\right)$, where $\lambda_{n}$ is the density scrape-off length and $d_{\text {gap }}$ the vacuumgap depth. In order to get physically relevant results, the mesh size should be sufficiently refined to capture the contribution of high wavenumbers waves propagating in the plasma. In case of LHRF antennas, high toroidal wavenumbers are due to the inevitable spurious lobes generated by LHRF phased array. In the case of the cold plasma in this range of frequencies, the medium wavelength decreases with increasing density. Thus, for a given convergence target criteria (for example in this work the maximum change in the magnitude of the scattering parameters between two consecutive passes is $\Delta_{S} \approx 10^{-3}$ ), the number of elements will increase with the initial edge density. Putting the plasma directly at the contact of the waveguide leads to high order modes (most being evanescent) at the interface between the antenna and the plasma. These modes requires very fine mesh, unless with the reflection inside the antenna would be generally overestimated. A vacuum gap region between the antenna and the plasma speedsup very much the convergence process since it attenuates these high order modes. This model required around 150000 elements for a convergence target of $\max \left|\Delta_{S}\right|<10^{-3}$, solved in 10-20 minutes on a 64 GB desktop computer. For comparison, ALOHA results are obtained in less than a minute.

The figures 2 illustrate the benchmark with the ALOHA code on the electric field and the power density spectrum calculated from the Fourier transform of the electric and magnetic fields at the mouth of the LHRF antenna. The maximum electric field in HFSS is in general lower than in ALOHA because of the smoothing effect of the mesh. The average value is however of the same order. The average Reflection Coefficient (RC) calculated from HFSS matches well ALOHA's calcula- tions when the edge density is higher than the cutoff density (Figure 3). Looking in details, waveguide per waveguide, HFSS results have a reduced $\mathrm{RC}$ amplitude excursion than ALOHA's.

\section{Conclusion}

The ANSYS HFSS software have been benchmarked for coupling calculations of Lower Hybrid Resonance Frequency to usual edge tokamak cold magnetize plasma conditions. Reflected power and electric field obtained in HFSS agree well with the dedicated LH coupling code ALOHA if absorbing boundary conditions are properly set. In practice, usual edge plasma scenario can be modelled with the requirement of a vacuum gap. The required memory resources increases with the electron density increase, which can be a practical limitation for high-density scenarios.

Since it is possible to define inhomogeneous media in HFSS, it is possible to describe equationbased evolution or to directly use numerical data (coming from edge plasma diagnostic such as reflectometers) to model various coupling scenarios. The results obtained can be used by antenna designers to evaluate coupling performances.

\section{References}

[1] T. H. Stix, Waves in Plasmas, Springer, New-York, 1992.

[2] J. Preinhaelter, J. Hillairet, D. Milanesio, R. Maggiora, J. Urban, L. Vahala, G. Vahala, Efficient full wave code for the coupling of large multirow multijunction LH grills, Nuclear Fusion 57 (11) (2017) 116060. doi: 10.1088/1741-4326/aa7f4f.

[3] J. Hillairet, D. Voyer, A. Ekedahl, M. Goniche, M. Kazda, O. Meneghini, D. Milanesio, M. Preynas, ALOHA: an Advanced LOwer Hybrid Antenna coupling code, Nuclear Fusion 50 (12) (2010) 125010. doi: 10.1088/0029-5515/50/12/125010.

[4] D. Milanesio, O. Meneghini, R. Maggiora, S. Guadamuz, J. Hillairet, V. Lancellotti, G. Vecchi, TOPLHA: an accurate and efficient numerical tool for analysis and design of LH antennas, Nuclear Fusion 52 (1) (2012) 013008. doi : 10.1088/0029-5515/52/1/013008.

[5] O. Meneghini, S. Shiraiwa, W. Beck, J. Irby, P. Koert, R. R. Parker, R. Viera, J. Wilson, S. Wukitch, V. Bobkov, J.-M. Noterdaeme, Integrated numerical design of an innovative Lower Hybrid launcher for Alcator C-Mod, in: AIP Conference Proceedings, Vol. 1187, 2009, pp. 423-426. doi:10.1063/1.3273783.

[6] S. Shiraiwa, J. C. Wright, P. T. Bonoli, T. Kolev, M. Stowell, RF wave simulation for cold edge plasmas using the MFEM library, EPJ Web of Conferences 157 (2017) 03048. doi:10.1051/epjconf/201715703048. 

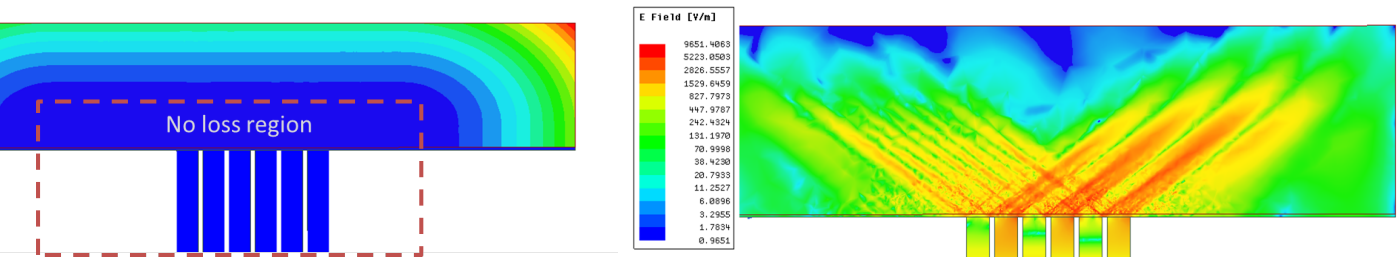

Figure 1: $x-z$ maps in the middle of the waveguide height. Left: conductivity map in the toroidal plane. Right: electric field magnitude (log scale).
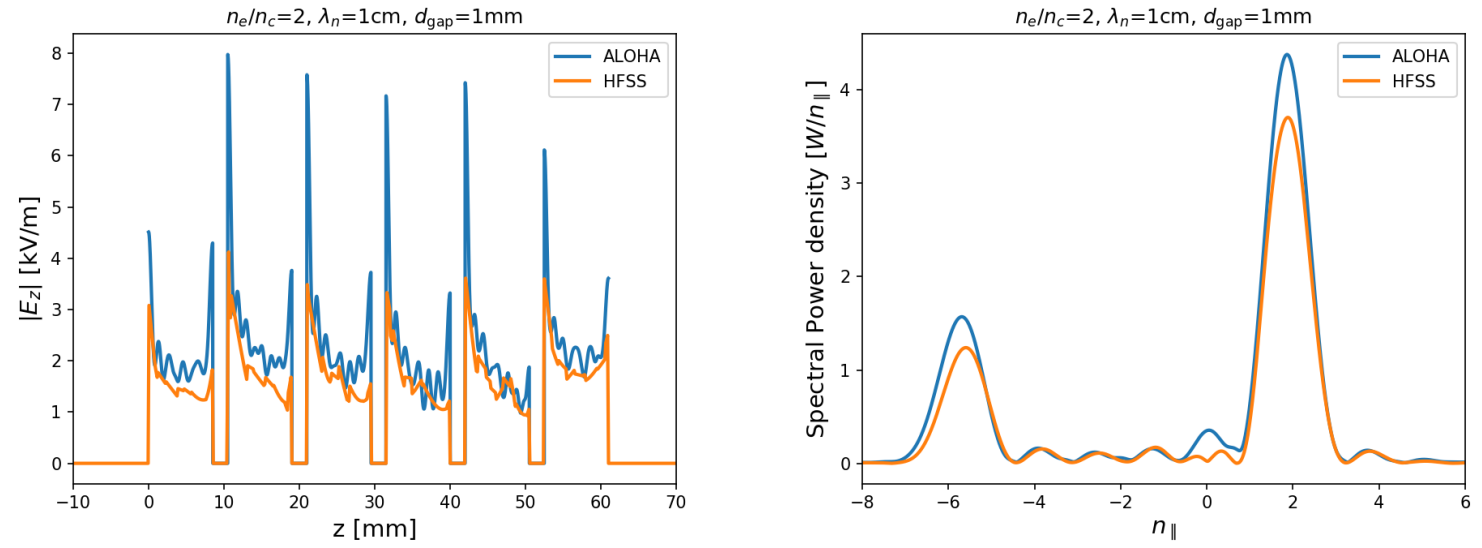

Figure 2: Example of comparison between ALOHA and HFSS $\left(n_{e}=2 n_{c}, \lambda_{n}=1 \mathrm{~cm}, 1 \mathrm{~mm}\right.$ vacuum gap). Left: toroidal component of the electric field at the mouth of the antenna. Right: Power density spectrum excited by the antenna.

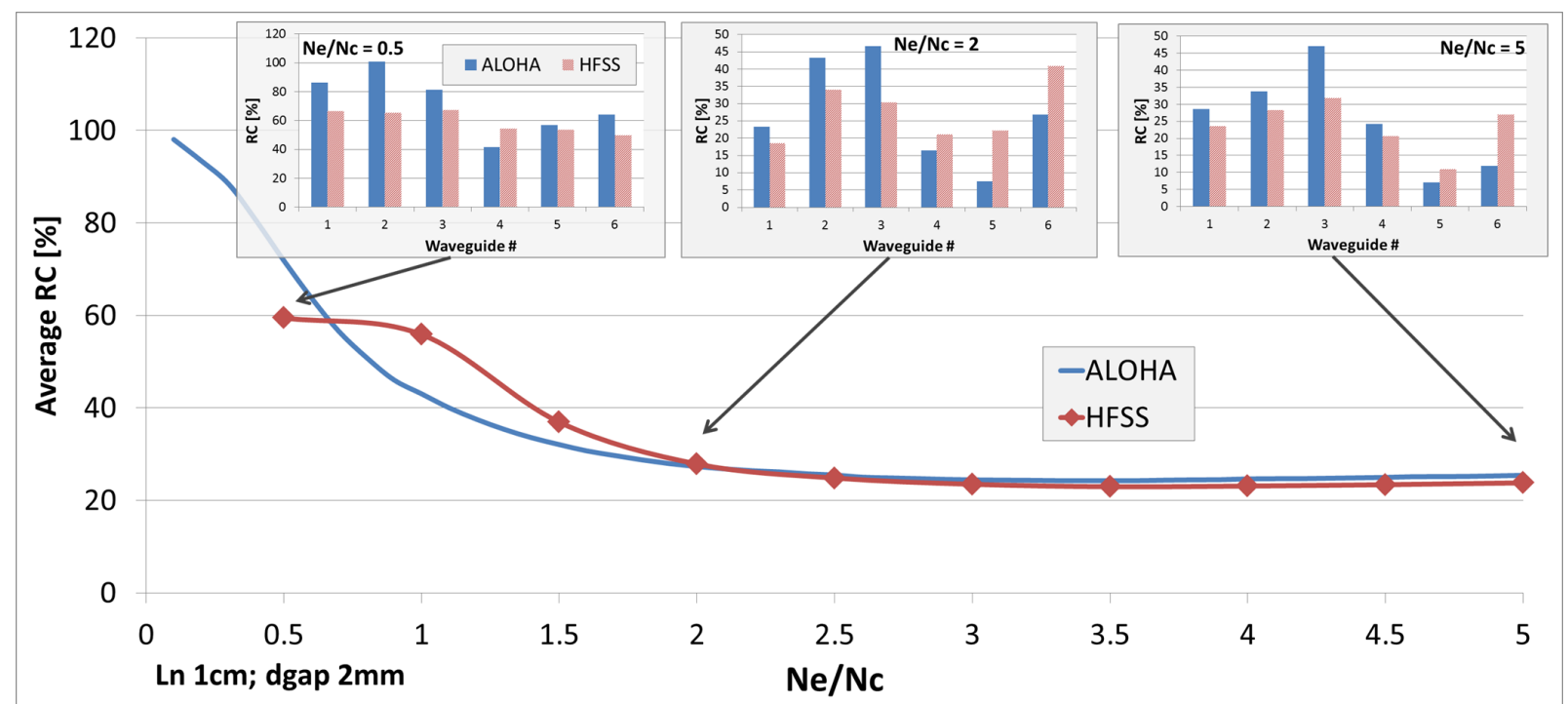

Figure 3: Reflection Coefficient (RC) as calculated by ALOHA and HFSS versus the edge density $\left(n_{e} / n_{c}\right)$ with $\lambda_{n}=1 \mathrm{~cm}$, $d_{\text {gap }}=2 \mathrm{~mm}$ vacuum gap. 
[7] J. Jacquot, L. Colas, F. Clairet, M. Goniche, S. Heuraux, J. Hillairet, G. Lombard, D. Milanesio, 2D and 3D modeling of wave propagation in cold magnetized plasma near the Tore Supra ICRH antenna relying on the perfecly matched layer technique, Plasma Physics and Controlled Fusion 55 (11) (2013) 115004. doi:10.1088/ 0741-3335/55/11/115004.

[8] E. Bécache, P. Joly, M. Kachanovska, Stable perfectly matched layers for a cold plasma in a strong background magnetic field, Journal of Computational Physics 341 (2017) 76-101. doi:10.1016/j.jcp.2017.03.051.

[9] A. F. Oskooi, L. Zhang, Y. Avniel, S. G. Johnson, The failure of perfectly matched layers, and towards their redemption by adiabatic absorbers, Optics Express 16 (15) (2008) 11376. doi:10.1364/OE.16.011376. 